\title{
There's a xeno in your future
}

Homer's chimaera was a 'fabulous' creature with the body of a goat, the head of a lion, and the tail of a dragon. What the poet did not think to add was the heart of a woman.

Nor did Homer envision a woman with the heart of a pig, or a man with the blood cells of a baboon - though each is likely to exist before this century is out. In fact, an AIDS patient in San Francisco hopes to have a transfusion of baboon bone marrow within months (the sooner the better). And veterinary scientists have for some time been busy creating transgenic pigs for human transplantation - the pig being an animal whose natural heart approximates the human heart in size.

It is now ordinary in the art and science of organ transplantation to think about the use of animals as spare parts for dying human beings. Indeed, it has been a dream for more than 30 years. In the 1960s a small, daring band of transplant pioneers gave xenotransplantation an experimental try with animal-to-animal and animal-to-human organ grafts. The failures were dramatic. A pig's heart was transplanted into a dog. Within 30 seconds, the dog was dead. A chimpanzee's heart was given to a man, who died on the operating table.

Meanwhile, surgeons interested in kidney transplants were also experimenting. The ethical climate in the early 1960s was right. Dialysis was not yet perfected, and cadaveric organs were virtually unavailable. The risks were worth it. People began thinking about baboons as organ donors. The more imaginative surgeons (perhaps those given to reading science fiction) predicted that one day every potential transplant recipient might have his own baboon. One limitation to this medical fantasy was that baboons come only in A, B and AB blood types. There is no $O$ or universal donor. On the other hand, they are more plentiful than chimpanzees. Chimps, who do have type $\mathrm{O}$ blood, were to be carefully bred and used selectively in medical transplantation.

It was altogether an exciting yet frustrating time. The principal barrier to success was not the operation (the surgeons had that down pat) but immunology. Transplanting an organ across species or 'xenotransplantation' (from the Greek for strange) failed because the human immune system rapidly rejected the potentially life-saving animal organ - the same problem that faced

*See pages 869-873, 964-966, 967-968 and 969. recipients of genetically unrelated human organs.

The immune barrier that once looked impenetrable fell as immunologists acquired drugs to protect human donor organs from rejection. Human-to-human organ transplantation began to thrive in the late 1970 s as individuals living on borrowed time with new hearts, kidneys, livers, lungs, and bone marrow became nearly commonplace in the annals of surgery and immunology. Xenotransplantation fell out of favour.

But the problem of supply versus demand has persisted ever since the early days. Throughout the world, literally thousands of patients wait in line for a donor organ that never comes. Cases such as that of baseball legend Mickey Mantle bring the issue vividly to public attention. With human donor livers in short supply, the Mantle case illustrates why animal-to-human transplantation is once again high on the research agenda.

As four papers* in this issue of Nature Medicine confirm, the central barrier to xenotransplantation is organ rejection - just as it was 30 years ago. But real progress is being made and there is even something of a consensus about how to tackle the problem of crossspecies immune rejection. The 'hyperacute rejection' or HAR that stands in the way of successful transplantation appears to be mediated by the complement complex of serum proteins, which can cause cell lysis in antigen-antibody reactions. Isolating and therapeutically overcoming the ability of complement to reject animal organs is now a research priority.

There are, of course, additional matters to be resolved - some physiological, others that fall into the realm of social ethics. The possibility that an animal organ might harbour a virus that could be spread throughout the population is among the more apocalyptic. But such scary visions should not stand in the way of carefully designed and monitored laboratory and human experimentation. Physicians have just receive final approval from the US Food and Drug Administration to transplant HIV-resistant baboon bone marrow into a young man dying of AIDS. The likely first recipient of baboon marrow has said it well (Nature Medicine 1, 729). "My doctors want to go forward with an experiment that may not save my life, but could well lead to a treatment that will save thousands of others. We can't let fears of theoretical risks stop research." Especially since there is no such thing as medical research that is without risk.

Barbara J. Culliton 